\title{
Amphetamine and Methamphetamine Increase NMDAR-GluN2B Synaptic Currents in Midbrain Dopamine Neurons
}

\author{
Ming-Hua Li', Suzanne M Underhill ${ }^{2}$, Cheryl Reed ${ }^{3}$, Tamara J Phillips ${ }^{3,4}$, Susan G Amara ${ }^{2}$ and \\ Susan L Ingram*,I \\ 'Department of Neurological Surgery, Oregon Health \& Science University, Portland, OR, USA; ${ }^{2}$ National Institute of Mental Health, National \\ Institutes of Health, Laboratory of Molecular and Cellular Neurobiology, Bethesda, MD, USA; ${ }^{3}$ Department of Behavioral Neuroscience, Oregon \\ Health \& Science University, Portland, OR, USA; ${ }^{4}$ VA Portland Health Care System, Portland, OR, USA
}

\begin{abstract}
The psychostimulants amphetamine (AMPH) and methamphetamine (MA) are widely abused illicit drugs. Here we show that both psychostimulants acutely increase NMDA receptor (NMDAR)-mediated synaptic currents and decrease AMPA receptor (AMPAR)/ NMDAR ratios in midbrain dopamine neurons. The potentiation depends on the transport of AMPH into the cell by the dopamine transporter. NMDAR-GluN2B receptor inhibitors, ifenprodil, RO 25-698I, and RO 04-5595, inhibit the potentiation without affecting basal-evoked NMDA currents, indicating that NMDAR-GluN2B receptors are activated by AMPH. A selective peptide inhibitor of AMPHdependent trafficking of the neuronal excitatory amino acid transporter 3 (EAAT3) blocks potentiation, suggesting that EAAT3 internalization increases extracellular glutamate concentrations and activates GluN2B-containing NMDARs. Experiments with the usedependent NMDAR blocker, MK-80 I, indicate that potentiated NMDARs reside on the plasma membrane and are not inserted de novo. In behavioral studies, GluN2B inhibitors reduce MA-mediated locomotor activity, without affecting basal activity. These results reveal an important interaction between dopamine and glutamatergic signaling in midbrain dopamine neurons in response to acute administration of psychostimulants.

Neuropsychopharmacology (2017) 42, I539-I547; doi:I0.I038/npp.2016.278; published online I8 January 2017
\end{abstract}

\section{INTRODUCTION}

Repeated use of the psychostimulants amphetamine (AMPH) and methamphetamine (MA) leads to long-term adaptations in the central nervous system, including locomotor sensitization (Karler et al, 1989; Kelly et al, 2008) that can be observed following a single injection of AMPH (Vanderschuren et al, 1999). The cellular mechanisms underlying sensitization are not well understood, but AMPH or MA administration is associated with increased dopamine and glutamate release in midbrain areas (Lominac et al, 2012; Vanderschuren et al, 1999; Wolf et al, 2000; Zhang et al, 2001), suggesting an intricate interplay between these two neurotransmitter systems in midbrain circuits. Indeed, locomotor sensitization can be blocked with NMDA receptor (NMDAR) antagonists (Karler et al, 1989; Yap et al, 2005), implying that NMDARs have an important role in synaptic plasticity induced by psychostimulants.

\footnotetext{
*Correspondence: Dr SL Ingram, Department of Neurological Surgery, Oregon Health \& Science University (OHSU), 3I 8I SW Sam Jackson Park Road, Portland, OR 97239, USA, Tel: 503494 I220, Fax: 503494 2664, E-mail: ingrams@ohsu.edu

Received 29 August 2016; revised 29 November 2016; accepted II December 2016; accepted article preview online 15 December 2016
}

AMPH and MA have multiple actions on dopamine neurons; they are substrates for the dopamine transporter (DAT) and vesicular monoamine transporter (Sulzer, 2011) and induce trafficking of DAT (Johnson et al, 2005; Kahlig et al, 2006; Saunders et al, 2000). We recently showed that AMPH also induces trafficking of the neuronal excitatory amino acid transporter 3 (EAAT3) from the plasma membrane of dopamine neurons (Underhill et al, 2014). This family of glutamate transporters includes five isoforms that regulate the temporal and spatial influence of glutamate at the synapse and protect neurons from glutamate excitotoxicity. In general, EAAT isoforms 1 and 2 are found predominantly in astrocytes, EAAT3 in neurons, EAAT4 in cerebellar Purkinje cells, and EAAT5 expression is restricted to the retina (Danbolt, 2001). EAAT3 trafficking increased NMDAR synaptic currents in mouse dopamine neurons localized to both the substantia nigra pars compacta $(\mathrm{SNc})$ and ventral tegmental area (Underhill et al, 2014). Here, we further explore the effects of $\mathrm{AMPH}$ and MA on glutamatergic synaptic currents in SNc dopamine neurons. We show that AMPH superfusion potentiates NMDAR-mediated synaptic currents and that the potentiation is dependent on the activation of NMDARs containing GluN2B subunits. Further, activation of NMDARGluN2B subunits contributes to locomotor stimulation induced by acute administration of MA. 


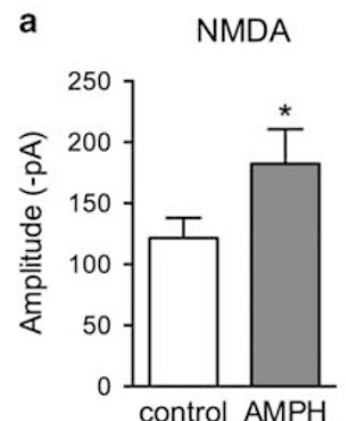

c

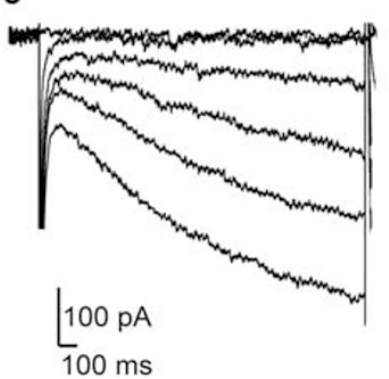

AMPA
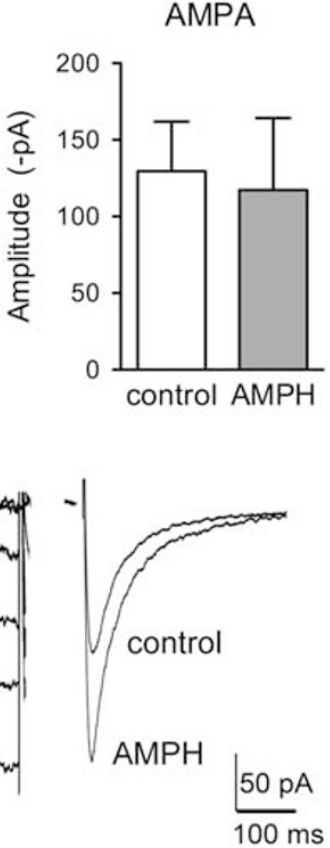

b
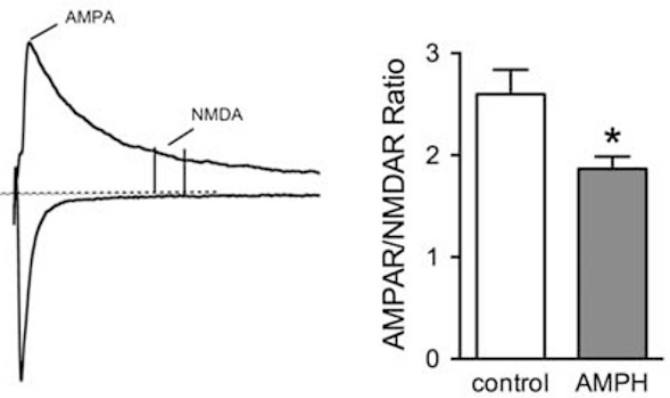

d

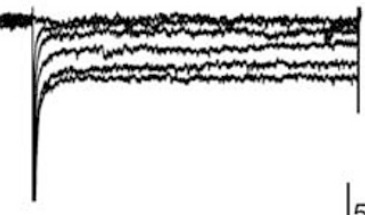

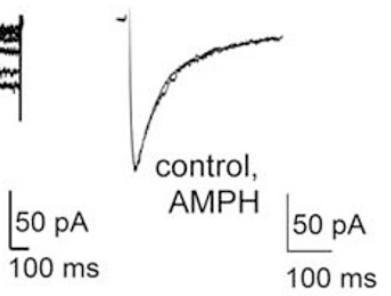

Figure I Amphetamine (AMPH) increases NMDA receptor (NMDAR) excitatory postsynaptic currents (EPSCs) in substantia nigra pars compacta (SNc) dopamine neurons. (a) Isolated NMDAR responses were significantly potentiated by AMPH $(n=14)$, but AMPA receptor (AMPAR) EPSCs were unaffected $(n=8)$. (b) To determine AMPAR/NMDAR ratios, evoked AMPAR and NMDAR EPSC amplitudes were measured at $+40 \mathrm{mV}$ at the peak and $40 \mathrm{~ms}$ later, respectively. AMPAR/NMDAR ratios were significantly decreased after superfusion of AMPH $(10 \mu M)$ for 20 min $(n=7)$. (c) The potentiation by AMPH was observed in cells expressing a hyperpolarization-activated current $\left(I_{h}\right)$. (d) No potentiation occurred in $I_{h}$ negative SNc neurons. * $p<0.05$.

\section{MATERIALS AND METHODS}

\section{Approvals}

All procedures were performed in strict accordance with the Guide for the Care and Use of Laboratory Animals as adopted and promulgated by the National Institutes of Health and approved by the Institutional Animal Care and Use Committees of Oregon Health \& Science University and the VA Portland Health Care System.

\section{In Vitro Studies}

Rats. Postnatal days 25-45 male Sprague-Dawley rats were used for all electrophysiology experiments. Midbrain horizontal slices were prepared and the electrophysiological and biochemical experiments performed as described previously (Underhill et al, 2014). Detailed methods and chemicals used are described in Supplementary Materials and Methods.

Data analysis. All data are reported as mean \pm SEM. Student's $t$-test and analysis of variance (ANOVA) were used where appropriate. The criterion for significance was set at $p<0.05$.

\section{Behavioral Study}

Adult (73-118 days old) male and female MA low drinking mice from the MA Abuse Research Center Animal Core within the VA Portland Health Care System animal facility were used for this study. Locomotor activity testing was performed on 3 consecutive days to allow for habituation to handling and injection (Day 1), collection of baseline activity data (Day 2), and collection of data after drug treatment (Day 3). RO 04-5595 (RO) HCl (Abcam, Cambridge, MA) and (+)MA HCl (Sigma, St Louis, MO) were dissolved in sterile physiological saline on the morning of the study. Activity tests were $60 \mathrm{~min}$ in duration and data were collected in $5 \mathrm{~min}$ periods in automated activity monitors (Accuscan; $20 \times 40 \times 40 \mathrm{~cm}^{3}, \mathrm{H} \times \mathrm{W} \times \mathrm{L}$ ), as described previously (Gubner and Phillips, 2015). For detailed methods and data analyses, see Supplementary Materials and Methods.

\section{RESULTS}

\section{AMPH Potentiates NMDAR-Mediated Synaptic Currents in Rat SNc Dopamine Neurons}

The acute effects of AMPH on excitatory neurotransmission were determined using whole-cell patch-clamp recordings from rat brain slices containing SNc dopamine neurons. NMDAR-mediated excitatory postsynaptic currents (EPSCs) were evoked at $-70 \mathrm{mV}$ and isolated in the presence of the AMPA receptor (AMPAreceptor) inhibitor NBQX $(10 \mu \mathrm{M})$ in nominally $\mathrm{Mg}^{2+}$-free extracellular solution. AMPH $(10 \mu \mathrm{M})$ potentiated evoked NMDAR EPSCs by $\sim 50 \%$ (control: $-122 \pm 16 \mathrm{pA}$; AMPH: $-182 \pm 28 \mathrm{pA} ; n=14$; paired $t$-test, $t_{(13)}=4.01, p=0.0015$; Figure 1a). AMPAR-mediated EPSCs were isolated at $-70 \mathrm{mV}$ in $\mathrm{Mg}^{2+}$-containing extracellular solution. AMPH did not significantly change the amplitudes of AMPAR-mediated EPSCs (control: $-130 \pm 32 \mathrm{pA}$; AMPH: $-117 \pm 47 \mathrm{pA} ; n=8$; paired $t$-test, $t_{(7)}=0.69, p=0.51$; Figure 1a). To further explore the effects 
a

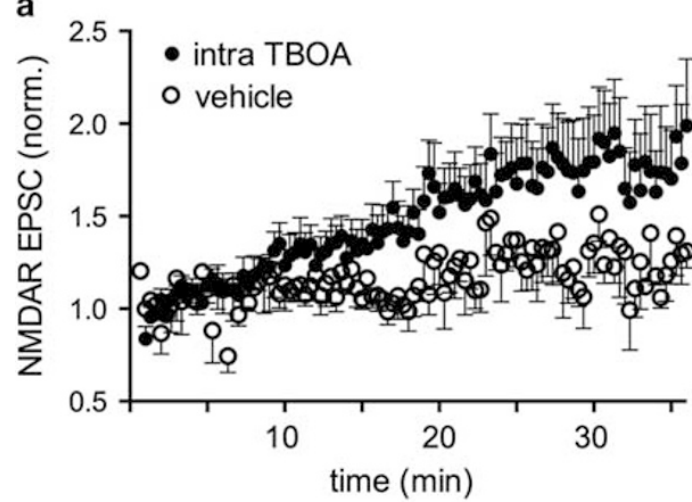

c

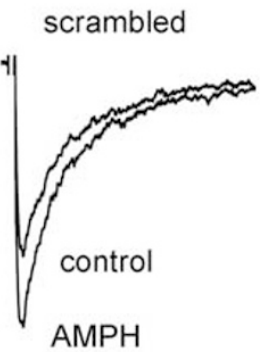

EAAT3 peptide

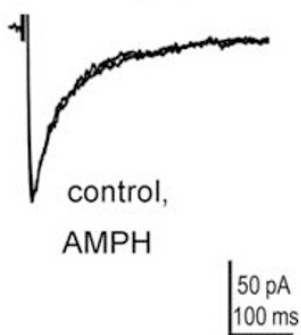

b

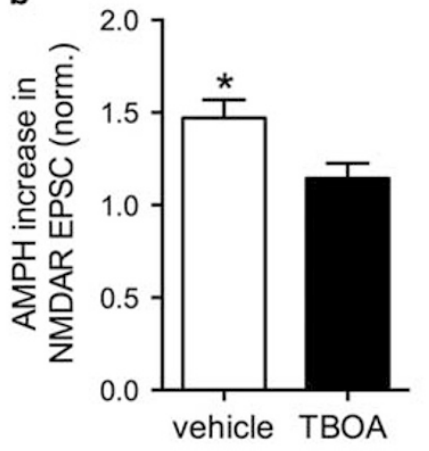

d

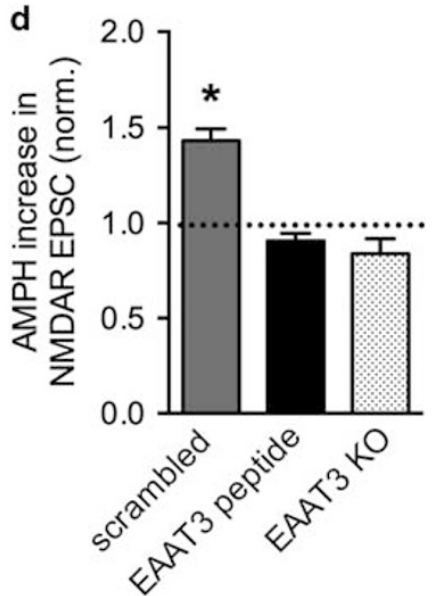

Figure 2 Excitatory amino acid transporter 3 (EAAT3) internalization is involved in the potentiation of NMDA receptor (NMDAR) excitatory postsynaptic currents (EPSCS) by amphetamine (AMPH). (a) Time course after breaking into whole-cell mode $(t=0$ min) with pipette solutions containing threo- $\beta$ benzyloxyaspartate (TBOA) (500 $\mu \mathrm{M} ; n=9)$ or vehicle control $(n=6)$. (b) Summary data normalized to baseline EPSCs (first 4 min) showing that intracellular TBOA occludes subsequent AMPH potentiation of NMDAR EPSCs $(* p<0.05)$. (c) Averaged NMDAR EPSCs with either the scrambled peptide or the EAAT3 inhibitor peptide in the whole-cell pipette solution. AMPH potentiation is blocked in cells recorded with the EAAT3 inhibitor peptide in the pipette solution. (d) Summary data normalized to baseline (first $4 \mathrm{~min}$ ) showing a significant potentiation of NMDAR EPSC amplitudes with the scrambled peptide $(n=4, * p<0.05)$, but no potentiation with the EAAT3 inhibitor peptide $(n=7)$. AMPH-mediated potentiation is absent in EAAT3 knockout $(K O)$ mice $(n=10)$.

of AMPH on the AMPAR/NMDAR ratio, EPSCs were evoked at $+40 \mathrm{mV}$ to measure AMPAR and NMDAR currents at the same voltage. Superfusion of AMPH $(10 \mu \mathrm{M})$ significantly decreased AMPAR/NMDAR ratios by $\sim 30 \%$ (control: $2.6 \pm 0.2$; AMPH: $1.9 \pm 0.1 ; n=7$; paired $t$-test, $t_{(6)}=3.87, p=0.008$; Figure 1b). The AMPH-induced potentiation of NMDAR currents was only observed in SNc neurons with a hyperpolarization-activated cation channel current $\left(I_{h}\right.$; Figures $1 \mathrm{c}$ and d), indicating that the effects of AMPH were expressed selectively in SNc dopamine neurons.

\section{AMPH-Mediated Potentiation of NMDAR EPSCs is Dependent on DAT}

We previously showed that AMPH-mediated potentiation in mouse DA neurons is dependent on DAT (Underhill et al, 2014). The DAT inhibitor GBR12909 blocks AMPHmediated potentiation in rat dopamine neurons (Supplementary Figure S1), indicating that AMPH-mediated modulation of NMDAR EPSCs depends on the binding and/or transport of AMPH by the DAT. All experiments were carried out in the presence of D1 and D2 receptor inhibitors to rule out possible effects of AMPH-mediated increases in extracellular dopamine levels. AMPH applied intracellularly also potentiated NMDAR EPSCs $(-289 \pm$ $24 \mathrm{pA}$ compared with baseline EPSCs $-133 \pm 12 \mathrm{pA}$, paired $t$-test, $t_{(4)}=7.53, p=0.002$ ), confirming that transport of AMPH into the cell is required for AMPH effects on NMDA signaling.

Midbrain dopamine neurons express the vesicular glutamate transporter 2 and have been shown to corelease glutamate (Chuhma et al, 2009; Stuber et al, 2010). We used paired-pulse experiments to confirm that the change in NMDAR EPSC amplitude was due to a change in postsynaptic NMDAR activation and not due to a change in presynaptic glutamate release. Paired-pulse ratios $(\mathrm{p} 2 / \mathrm{p} 1)$ were not different in the absence $(0.6 \pm 0.1)$ or presence of AMPH $(0.7 \pm 0.1, n=6$, paired $t$-test, $\left.t_{(5)}=0.95, p=0.38\right)$, providing evidence that potentiation of EPSCs by AMPH occurs via modulation of postsynaptic NMDARs. 


\section{EAAT3 Trafficking is Involved in NMDAR EPSC Potentiation by AMPH}

AMPH-induced trafficking of DAT from the plasma membrane is well established (Saunders et al, 2000) and depends on AMPH transport into dopamine neurons through the DAT (Kahlig et al, 2006; Wheeler et al, 2015). In addition, we recently reported that both the DAT and a neuronal glutamate transporter, EAAT3, are trafficked from the membrane in response to AMPH in mouse dopamine neurons (Underhill et al, 2014). To investigate the role of glutamate transporters in the actions of AMPH on NMDAR EPSCs, the non-transportable EAAT inhibitor TBOA $(500 \mu \mathrm{M})$ was added to the pipette solution in whole-cell patch-clamp recordings from DA neurons in the SNc brain slice preparation. Evoked NMDAR EPSCs increased over the first $30 \mathrm{~min}$ when exposed to intracellular TBOA, compared with vehicle (Figure 2a), indicating that selective blockade of EAAT3 in the recorded cell is sufficient to observe enhanced NMDAR EPSCs. NMDAR EPSCs were enhanced with intracellular TBOA by $145 \pm 13 \%(n=9)$ compared with intracellular vehicle $\left(105 \pm 8 \%, n=6, t_{(13)}=2.37, p=0.03\right)$. The potentiation by AMPH was significantly occluded in the presence of intracellular TBOA $(14 \pm 8 \%$ increase, $n=6)$, compared with vehicle controls $\left(47 \pm 10 \%, n=6, t_{(10)}=2.52\right.$, $p=0.03$; Figure $2 \mathrm{~b}$ ).
We reported previously that trafficking of EAAT3 by AMPH is disrupted by a specific peptide targeted to amino acids in the $\mathrm{C}$ terminus of EAAT3 that are involved in EAAT3 endocytosis from the plasma membrane (Underhill et al, 2014). Incorporation of the EAAT3 blocking peptide into the intracellular recording solution blocked the ability of AMPH to potentiate NMDAR EPSCs (EAAT3 inhibitor peptide: $-9 \pm 4 \%(n=7)$ compared with scrambled peptide: $43 \pm 6 \%\left(n=4 ; t_{(9)}=7.59, p<0.0001\right.$; Figures $2 c$ and $\left.d\right)$. These data provide evidence that potentiation of evoked NMDAR EPSCs is dependent on AMPH-induced trafficking of EAAT3. To further test the role of EAAT3 in the effects of AMPH, we examined the ability of AMPH to potentiate NMDAR responses in EAAT3-knockout mice and found no potentiation $(-16 \pm 8 \%$; one-sample $t$-test, $p=0.07$, $n=10)$.

EAAT3 transport activity is voltage dependent and is significantly decreased at positive membrane voltages typically used for measuring evoked NMDAR currents (+40 mV; Wadiche et al, 1995). Thus, the effects of AMPH and MA may be underestimated when measuring currents at $+40 \mathrm{mV}$. Indeed, we observed a decreased effect of $\mathrm{AMPH}$ at $+40 \mathrm{mV}$ in both rats and mice (Supplementary Figure S2). To reduce this confound, recordings were carried out at $-70 \mathrm{mV}$ in nominally $\mathrm{Mg}^{2+}$-free extracellular solution.
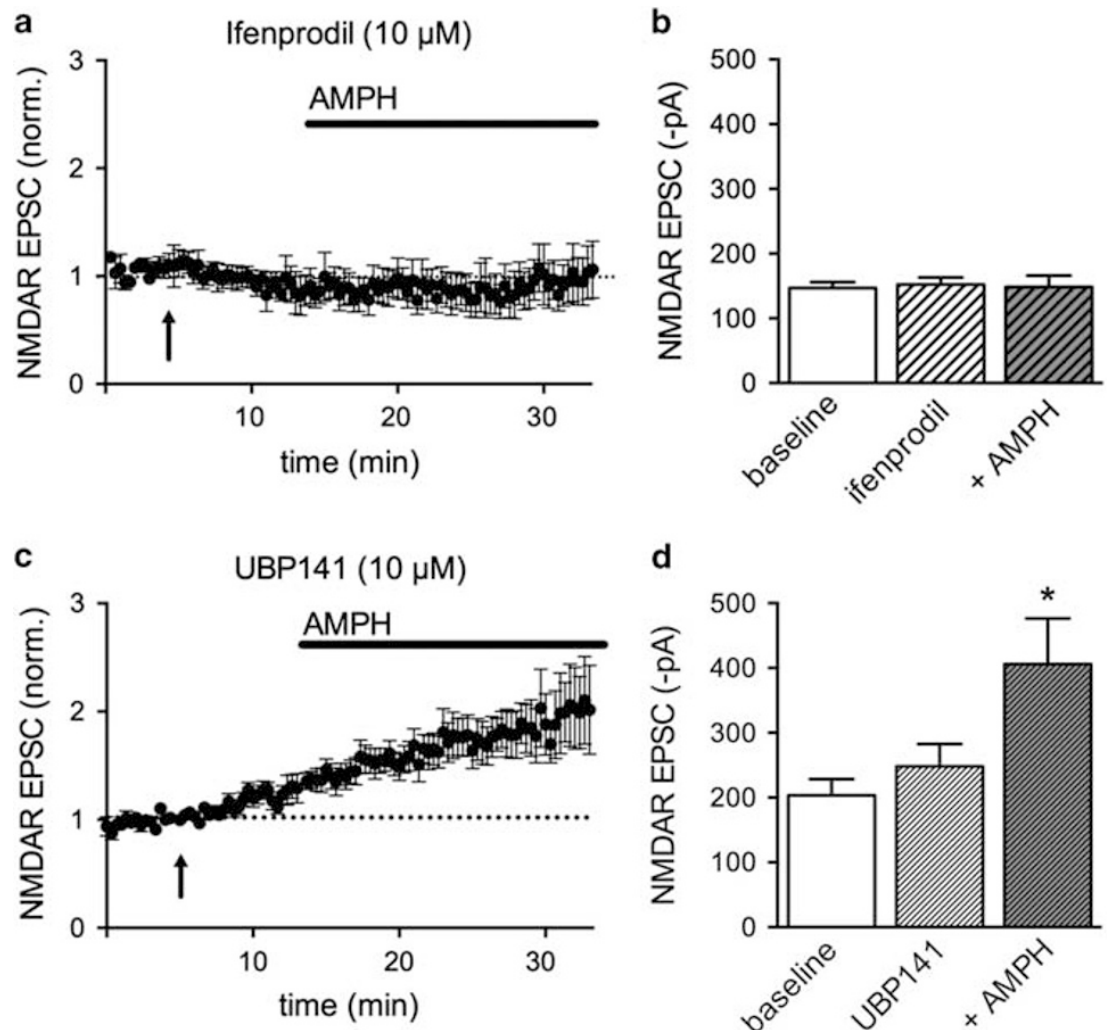

Figure 3 Amphetamine (AMPH) activates NMDA receptors (NMDARs) with GluN2B subunits. (a) Time course of evoked excitatory postsynaptic currents (EPSCs) in the absence and presence of the GluN2B subunit inhibitor ifenprodil ( 0 HM, applied at arrow) and after AMPH. Ifenprodil has no effect on basal NMDAR EPSCs but blocks potentiation by AMPH. Dotted line denotes baseline EPSCs. (b) Summary data of NMDAR EPSC amplitudes for baseline, ifenprodil, and ifenprodil+AMPH conditions. (c) Same experiment as in (a), using the NMDAR GluN2D-selective inhibitor UBPI4I (I0 HM). Dotted line denotes baseline EPSCs. (d) Summary data showing a significant increase in NMDAR EPSC amplitudes in the presence of UBPI4I+AMPH, compared with UBPI4I alone (repeated-measures one-way analysis of variance (ANOVA), Bonferroni post hoc test, ${ }^{*}<<0.05$ ). 
a
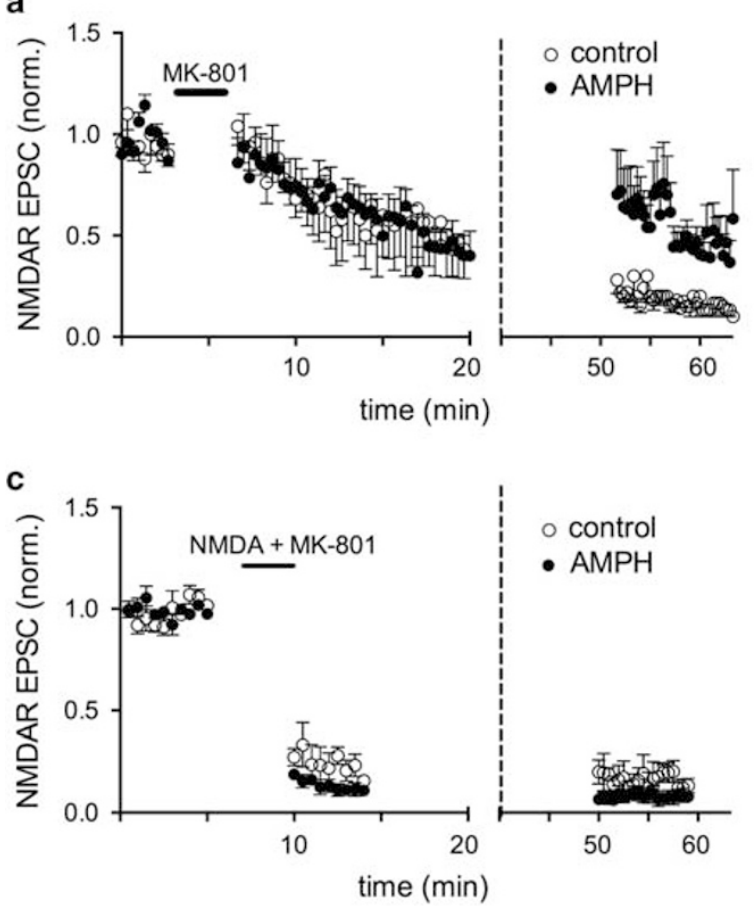

b
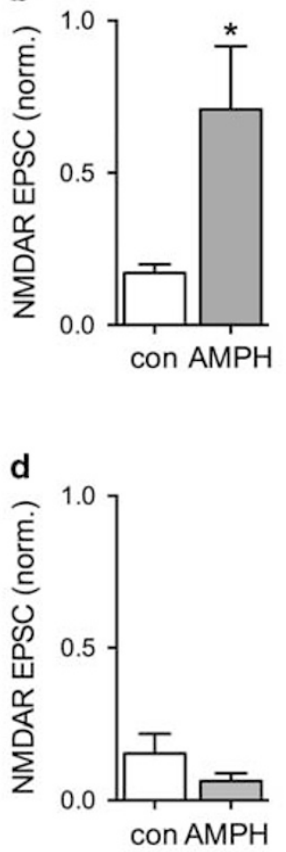
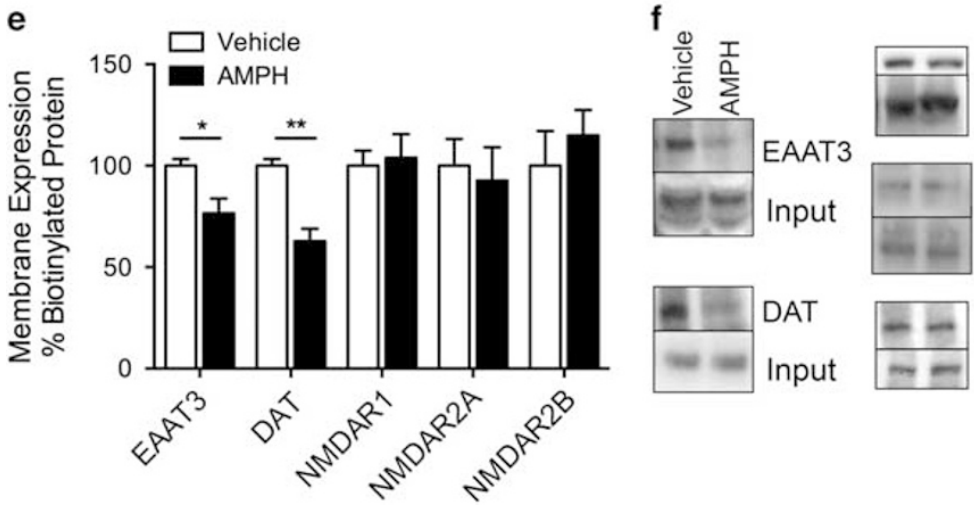

NMDAR1

Input

NMDAR2A

Input

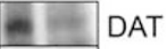

Input

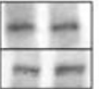

NMDAR2B

Input

Figure 4 Amphetamine (AMPH) activates NMDA receptors (NMDARs) that are not activated with evoked stimuli. (a) Synaptic NMDARs were blocked with MK-80 I ( $40 \mu \mathrm{M})$ superfusion and normal bipolar stimulation at $0.033 \mathrm{~Hz}$. Following $>20$ min of washout, AMPH (I $\mathrm{\mu M}$ ) was applied to half of the slices (dashed vertical line). NMDAR excitatory postsynaptic currents (EPSCs) in the presence of AMPH were larger compared with EPSCs in the absence of AMPH. Note split $X$ axis with expanded time course from 0 to 20 min to show cumulative block during synaptic stimulation. (b) Summary data comparing normalized

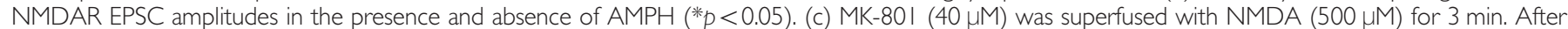
recovery of large NMDAR-mediated inward currents, bipolar stimulation at $0.033 \mathrm{~Hz}$ was initiated to monitor the extent of block by MK-80 In the presence of NMDA. AMPH did not increase NMDAR EPSCs following 40 min of washout of NMDA and MK-80I (dashed vertical line; $n=5$ ), compared with control $(n=3)$. (d) Summary data comparing normalized NMDAR EPSC amplitude following superfusion of AMPH. (e) Substantia nigra pars compacta (SNc) slices were incubated in vehicle or AMPH (IO MM) for $30 \mathrm{~min}$ and cell-surface proteins were labeled with a cell-impermeable biotinylation reagent. AMPH reduced biotinylated EAAT3 and DAT proteins $\left(n=4 ;{ }^{*} p<0.05\right.$, $\left.{ }^{*} *<0.00 \mathrm{I}\right)$. Surface biotinylated NMDA, R2A (GluN2A), and R2B (GluN2B) proteins in rat midbrain slices containing the SNc were not altered by incubation in AMPH for 30 min $(n=4)$. ( $f$ ) Representative western blots from biotinylation experiments.

\section{NMDARs Containing GluN2B Subunits are Involved in Potentiation of NMDAR Synaptic Transmission by AMPH}

Previous studies have shown that NMDARs containing GluN2B subunits have an important role in adaptations induced by cocaine and heroin (Liu et al, 2006; Shen et al, 2011). SNc dopamine neurons differentially express both GluN2B and GluN2D subunits during postnatal development (Brothwell et al, 2008); thus, we were interested in determining which subtypes of NMDARs were modulated by AMPH.

The GluN2B-selective inhibitor ifenprodil $(1 \mu \mathrm{M})$ did not affect baseline NMDAR EPSC amplitudes $(-117 \pm 8 \mathrm{pA}$ in ifenprodil compared with baseline $-116 \pm 9 \mathrm{pA}, n=4)$, but this concentration of ifenprodil blocked potentiation by AMPH $(-120 \pm 11 \mathrm{pA})$. We also used ifenprodil $(10 \mu \mathrm{M})$ and observed no effect on baseline NMDAR EPSCs $(-152 \pm 11 \mathrm{pA}$ in ifenprodil compared with baseline 
$-147 \pm 9 \mathrm{pA}, n=12)$, but this concentration also blocked potentiation by AMPH $(-148 \pm 18 \mathrm{pA}$; Figures $3 \mathrm{a}$ and $\mathrm{b})$. Similar results were obtained using another GluN2B-selective inhibitor, Ro 25-6981 (5 $\mu \mathrm{M})$ where NMDAR EPSC amplitudes were $-135 \pm 17 \mathrm{pA}$ compared with $-130 \pm 22 \mathrm{pA}$ $(n=6)$ in the presence of AMPH. These results indicate that GluN2B subunits are necessary for potentiation of NMDAR currents by AMPH.

The selective inhibitor of GluN2D subunits UBP141 $(10 \mu \mathrm{M})$ did not block AMPH-mediated potentiation of NMDAR-mediated currents (Figures $3 c$ and d). UBP141 did not significantly affect basal NMDAR EPSCs (control: $204 \pm 25$ pA compared with UBP141: $248 \pm 35$ pA), and NMDAR EPSCs were significantly increased in the presence of AMPH $(406 \pm 70 \mathrm{pA}$ repeated-measures one-way ANOVA, drug factor, $\left.\mathrm{F}_{(2,18)}=15.92, p=0.0001\right)$. A selective GluN2A subunit inhibitor TCN 201 (3 and $10 \mu \mathrm{M}$ ) did not block basal NMDAR EPSCs, but occluded the effects of AMPH (Supplementary Figure S3A). Similarly, R-CPP, an inhibitor reported to be selective for NMDAR-GluN2A subunits at low nanomolar concentrations (50 nM) and nonselective at higher concentrations $(2 \mu \mathrm{M})$, also occluded the effects of AMPH (Supplementary Figure S3B and C). However, R-CPP (50 nM) and TCN 201 induced trends toward increased NMDAR EPSC amplitude in the absence of $\mathrm{AMPH}$, thus it is difficult to interpret the lack of effect of AMPH. It is possible that the potentiated receptors are triheteromeric receptors that contain GluN2A and GluN2B subunits (Tovar et al, 2013). In either case, it is clear that AMPH induces incorporation of NMDAR-GluN2B subunits into the synapse, or alternatively, AMPH promotes glutamate spillover to extrasynaptic NMDARs containing GluN2B subunits.

\section{AMPH may cause Trafficking of Extrasynaptic NMDARs to the Synapse}

NMDARs can traffic to synapses from intracellular or extrasynaptic sites (Tovar and Westbrook, 2002). In an attempt to determine whether NMDARs containing GluN2B subunits are located within the synapse, we took advantage of the fact that the NMDA inhibitor MK-801 is an activity-dependent blocker with a very slow off-rate (Rosenmund et al, 1993). MK-801 $(40 \mu \mathrm{M})$ was superfused over the slice for $3 \mathrm{~min}$ at $-70 \mathrm{mV}$ while stimulating EPSCs and blocked receptors that were activated by synaptic release of glutamate. During washout of MK-801, stimulation was stopped and AMPH was applied to half of the slices for the last $10 \mathrm{~min}$ of the washout period before resuming stimulation of evoked EPSCs. Evoked NMDAR EPSCs were larger in the slices exposed to AMPH compared with control slices without AMPH (Figures 4a and b). In control slices, stimulation elicited NMDAR EPSCs that were $\sim 20 \%(n=6)$ of the initial amplitude, but NMDAR EPSCs were $\sim 70 \%(n=8)$ of the initial amplitude in slices exposed to AMPH $\left(t_{(12)}=2.21\right.$, $p=0.04)$. These results indicate that AMPH induces activation of NMDARs that are new to the synapse. These receptors could be receptors inserted de novo from an intracellular pool or a separate pool of extrasynaptic receptors.

To test the possibility that extrasynaptic receptors localized to the membrane were trafficking to the synapse, we repeated the experiment in Figure 4a in the presence of both NMDA and MK-801. NMDA $(500 \mu \mathrm{M})$ activates both synaptic and extrasynaptic NMDARs so that coapplication of NMDA $+\mathrm{MK}-801$ blocks all activated receptors at the plasma membrane. We confirmed the block of NMDA-stimulated receptors by monitoring the inward current induced by NMDA in addition to monitoring blockade of the evoked NMDAR EPSCs. NMDA induced large inward whole-cell currents (data not shown). When the NMDA-induced inward current returned to baseline following blockade by MK-801, evoked EPSCs were stimulated for $\sim 5 \mathrm{~min}$ to further block synaptic NMDARs (Figures $4 \mathrm{c}$ and d). Again, AMPH was applied to half of the slices after a washout period of $40 \mathrm{~min}$ in the absence of MK-801. NMDAR EPSCs in both control $(15 \pm 6 \%, n=3)$ and AMPH-treated slices $\left(6 \pm 3 \%, n=5\right.$, $t$-test, $\left.t_{(6)}=1.559, p=0.17\right)$ were similarly blocked, indicating that AMPH primarily affects NMDARs that are already on the plasma membrane. Further support for this interpretation came from surface biotinylation studies. AMPH did not alter the number of surface NMDA NR1, GluN2A, and GluN2B subunit proteins as measured by surface biotinylation studies in slices containing the SNc (Figures $4 \mathrm{e}$ and $\mathrm{f}$ ). As a positive control, we verified that trafficking of DAT and EAAT3 was stimulated by AMPH in rat dopamine neurons. The surface levels of DAT and

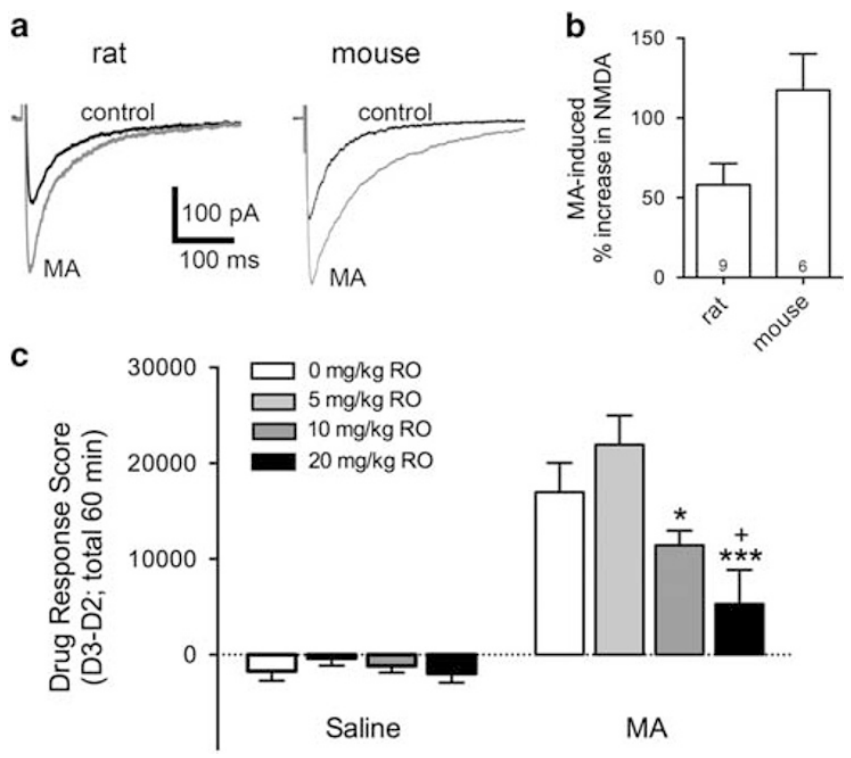

Figure 5 NMDA receptor-GluN2B (NMDAR-GluN2B) receptors mediate acute methamphetamine (MA) locomotor stimulation. (a) Representative traces recorded from rat and mouse dopamine neurons held at $-70 \mathrm{mV}$ in the absence and presence of MA $(10 \mu \mathrm{M})$. (b) Summary data showing that MA increased NMDAR excitatory postsynaptic currents (EPSCs) in both species. (c) Locomotor stimulation was measured for 60 min following pretreatment with different doses of RO 04-5595 (RO; intraperitoneally) given $30 \mathrm{~min}$ before injections of saline or MA $(2 \mathrm{mg} / \mathrm{kg}$, intraperitoneally); ( $n=9-16$ per RO group per MA group with 4-9 per sex in each group). Locomotor activity data after drug treatment were corrected for differences in basal activity level to create a Drug Response (D3-D2) Score. RO had no effect on activity level when given before saline treatment, but dose-dependently decreased MA-induced locomotor activity $(* p=0.03$ compared with $5 \mathrm{mg} / \mathrm{kg} \mathrm{RO}$; **** $p=0.00$ I compared with $5 \mathrm{mg} / \mathrm{kg} \mathrm{RO}$; $p=0.04$ compared with $0 \mathrm{mg} / \mathrm{kg} \mathrm{RO})$. 
EAAT3 were reduced by $37 \pm 6 \%\left(t_{(6)}=5.35, p=0.002\right)$ and $23 \pm 7 \%\left(t_{(6)}=2.95, p=0.03\right)$, respectively, following $\mathrm{AMPH}$ treatment. In addition, AMPH did not significantly affect rise and decay kinetics of the NMDAR synaptic currents, but there was a trend toward an increase in decay kinetics (Supplementary Figure S4). Taken together, these results support the idea that GluN2B-containing NMDARs are localized to extrasynaptic sites on the membrane and potentially move laterally into the synapse.

\section{GluN2B Inhibitors Reduce MA-Induced Locomotor Stimulation}

To test the relevance of GluN2B signaling in psychostimulant-induced behavior, we examined the ability of the water-soluble, selective GluN2B inhibitor RO 04,5595 (RO) to inhibit MA-induced locomotor stimulation in mice. MA $(10 \mu \mathrm{M})$ potentiates NMDA synaptic currents to a similar extent as AMPH (Figures $5 \mathrm{a}$ and $\mathrm{b}$ ). Mice were pretreated with different doses of $\mathrm{RO}$ or saline (intraperitoneally) $30 \mathrm{~min}$ before injections of saline or $\mathrm{MA}(2 \mathrm{mg} / \mathrm{kg}$, intraperitoneally). Baseline activity data were collected $24 \mathrm{~h}$ earlier under the same injection and handling conditions, when only saline was injected. Locomotor activity data after drug treatment were corrected for differences in baseline activity and analyzed by three-way ANOVA $(\operatorname{sex} \times \mathrm{RO}$ dose $\times$ MA dose). There were no trends toward significant main or interaction effects involving sex (all ps>0.47); therefore, data for the sexes were combined and analyzed by two-way ANOVA for the effects of RO dose and MA dose. There was a significant $\mathrm{RO}$ dose $\times$ MA dose interaction $\left(\mathrm{F}_{(3,94)}=3.41 ; p=0.02\right)$ and subsequent analyses identified significant RO dose effects within the MA-treated $(p<0.001)$, but not saline-treated mice. Post hoc comparisons for the effect of RO dose within the MA groups revealed that the $20 \mathrm{mg} / \mathrm{kg}$ RO group activity level was significantly lower than the activity levels of MA group mice treated with saline or $5 \mathrm{mg} / \mathrm{kg}$ RO. The activity level of the $10 \mathrm{mg} / \mathrm{kg}$ group was also significantly lower than the activity of the $5 \mathrm{mg} / \mathrm{kg}$ group (Figure 5c). These data indicate that NMDA-GluN2B receptors do not impact basal locomotor activity, but contribute to MA-stimulated locomotor activity.

\section{DISCUSSION}

The data presented here demonstrate that amphetamines potentiate glutamatergic neurotransmission in midbrain dopamine neurons through internalization of the neuronal glutamate transporter EAAT3 and subsequent activation of NMDARs containing GluN2B subunits. Inhibitors of GluN2B block AMPH and MA-mediated potentiation of NMDAR synaptic currents and locomotor stimulation. These data expand our understanding of the impact of regulated trafficking of neurotransmitter transporters described in our earlier paper (Underhill et al, 2014).

Psychostimulants increase both extracellular dopamine (Robertson et al, 1991; Sharp et al, 1987) and glutamate (Wolf et al, 2000; Zhang et al, 2001) concentrations in the midbrain. AMPH and MA increase extracellular dopamine levels by competing with dopamine for DAT, inhibiting forward DAT activity, internalizing the DAT, and altering vesicular dopamine uptake (Kahlig et al, 2006; Sulzer and Rayport, 1990), whereas the mechanism underlying the increase in glutamate is not as well understood. Recently, we described a mechanism for increased extracellular glutamate by showing evidence for AMPH-induced trafficking of the neuronal glutamate transporter EAAT3 from the plasma membrane of mouse dopamine neurons (Underhill et al, 2014). Here, we report that surface levels of DAT and EAAT3 are decreased by AMPH in midbrain slices from rats, resulting in potentiated NMDAR synaptic responses and decreased AMPAR/NMDAR ratios in $\mathrm{SNc}$ dopamine neurons.

Our studies focus on glutamate synaptic inputs onto midbrain dopamine neurons. Only neurons with pronounced hyperpolarization-activated $\left(I_{\mathrm{h}}\right)$ currents, typically identified as dopaminergic neurons in the SNc, responded to $\mathrm{AMPH}$ in the rat SNc. Potentiation of NMDAR currents was unaffected by superfusion of dopamine receptor inhibitors, or $\alpha 1$-adrenergic receptor inhibitors, suggesting that increased extracellular dopamine and activation of dopamine receptors do not contribute to the AMPH-mediated potentiation of synaptic currents. Instead, we found that the potentiation was blocked by the selective DAT inhibitor GBR12909, providing further support that AMPH effects are selective for dopamine neurons. As GBR12909 is not transported into the cell but blocks the actions of AMPH, these data are in agreement with a model in which AMPH is transported into dopamine neurons through DAT to initiate intracellular signaling events resulting in DAT and EAAT3 internalization (Underhill et al, 2014).

Many studies have focused on chronic administration of AMPH and MA and resulting locomotor sensitization. These studies show that behavioral sensitization is associated with an increase in AMPAR/NMDAR ratios $>12 \mathrm{~h}$ following $\mathrm{AMPH}$ and cocaine administration in vivo (Argilli et al, 2008; Borgland et al, 2004; Faleiro et al, 2004; Zhang et al, 1997) and the long-term potentiation of signaling by dopamine neurons within the SNc and VTA. Moreover, at these later times after administration, AMPH and cocaine induce changes in AMPAR currents with little or no change in NMDAR currents. Interestingly, both the potentiation of AMPAR signaling (Kalivas and Alesdatter, 1993; Ungless et al, 2001) and the sensitization of locomotor activity by AMPH appear to require activation of NMDARs (Karler et al, 1989; Vezina and Queen, 2000). Our data presented here demonstrate that the acute effects of $\mathrm{AMPH}$ and MA do involve changes in NMDAR currents, particularly those receptors containing GluN2B subunits and further support the idea that NMDAR activation may be prerequisite to longer term modulation of synaptic AMPAR signaling. Additional studies will be required to ascertain whether AMPH-mediated activation of NMDARs is an obligatory step that precedes AMPAR insertion into the synapse following in vivo administration of AMPH.

\section{AMPH Activates NMDARs Containing GluN2B Subunits}

NMDARs are tetramers primarily composed of NR1 subunits that bind the coagonist glycine, and NR2 subunits (including A and B) that bind glutamate (Cull-Candy et al, 2001). Dopamine neurons in the SNc preferentially express GluN2B and GluN2D subunits that form NMDARs 
comprised of two NR1 subunits with one GluN2B and one GluN2D subunit by postnatal day 21 (Brothwell et al, 2008). Brothwell et al (2008) previously showed that ifenprodil, the selective inhibitor of NMDARs containing GluN2B subunits, blocked $\sim 70 \%$ of the NMDAR EPSCs in SNc dopamine neurons at postnatal day 7 , but that the inhibitory effect of ifenprodil decreases significantly over development, suggesting that ifenprodil is less effective at blocking receptors containing one GluN2B subunit. Consistent with their results, we observed that ifenprodil had little effect on basal NMDAR EPSCs at ages greater than postnatal day 25. There also was no effect of UBP141, the selective GluN2D subunit inhibitor, on basal NMDAR EPSCs. However, ifenprodil, but not UBP141, effectively blocked AMPH-mediated potentiation of NMDAR-EPSCs.

GluN2B-containing NMDA receptors exhibit prolonged channel open times and greater overall $\mathrm{Ca}^{2+}$ current per event than receptors with other subunit conformations (Cull-Candy et al, 2001; Sobczyk et al, 2005). They are also critical for many forms of synaptic plasticity and learning/ memory (Barria and Malinow, 2002) and behavioral sensitization to drugs of abuse (Brown et al, 2011; Mao et al, 2009; Schilstrom et al, 2006; Schumann et al, 2009; Shen et al, 2011). Activation of NMDARs drives the transition from tonic to burst firing of DA neurons known to be important for conditioned responses to cues and long-term potentiation in midbrain dopamine neurons (Chergui et al, 1994; Harnett et al, 2009; Overton and Clark, 1997). Thus, AMPH-induced activation of NMDARs containing GluN2B subunits may be critical for conditioned responses. AMPH withdrawal (14 days), however, is associated with decreased GluN2B levels on the plasma membrane in the striatum (Mao et al, 2009), suggesting that activation of these receptors is transient. Alternatively, there may be important differences in trafficking in different brain areas or during withdrawal periods following induction of AMPH sensitization.

The exact location of the NMDARs activated by AMPH is unknown. The results of our experiments with MK-801 provide evidence that $\mathrm{AMPH}$ stimulates a population of extrasynaptic NMDARs containing GluN2B subunits or that it causes GluN2B-containing receptors to move laterally into the synapse. AMPH-mediated downregulation of EAAT3 could lead to increased glutamate levels in the synapse and spillover to extrasynaptic glutamate receptors or, alternatively, decreased glutamate clearance by EAAT3 outside the synapse could potentiate extrasynaptic receptor activation. It has not been established whether EAAT3 resides within synaptic specializations or within extrasynaptic domains of dopamine neurons. Extrasynaptic GluN2B-containing NMDARs have been described (Tovar and Westbrook, 1999) and it has been previously demonstrated that EAAT3 modulates extrasynaptic glutamate levels and activation of NMDARs containing GluN2B subunits in the hippocampus (Scimemi et al, 2009). One intriguing possibility is that $\mathrm{AMPH}$ activates silent synapses in the SNc (Kerchner and Nicoll, 2008). Silent synapses contain functional NMDARs but no functional AMPARs (Isaac et al, 1995), but activation of NMDARs in these nascent synapses is required for insertion of AMPARs (Brown et al, 2011; Huang et al, 2009). Increased numbers of silent synapses have been observed following induction of cocaine sensitization (Brown et al, 2011; Koya et al, 2012). However, these studies provide evidence for de novo insertion of NMDARs in the plasma membrane of nucleus accumbens neurons (Huang et al, 2009), whereas our data from experiments using MK-801 blockade in the presence of NMDA suggest that the NMDARs containing GluN2B subunits are already expressed on the membrane in the SNc.

In conclusion, these studies demonstrate that AMPH potentiates glutamatergic synaptic transmission via stimulation of EAAT3 internalization and activation of a new population of NMDARs containing GluN2B subunits. These data provide a novel cellular mechanism linking dopamine and glutamate neurotransmission in response to $\mathrm{AMPH}$ and MA in midbrain dopamine neurons responsible for the initial locomotor stimulating response to psychostimulants.

\section{FUNDING AND DISCLOSURE}

Research was funded by National Institutes of Health: National Institute of Drug and Alcohol Abuse (DA027625 (to SLI) and DA018165 (to TJP)), National Institute of Mental Health Intramural Program (MH002946 (to SGA)), and the Department of Veterans Affairs (to TJP). The authors declare no conflict of interest.

\section{ACKNOWLEDGMENTS}

We thank Katherine Suchland for excellent technical support and critical review of the manuscript.

\section{REFERENCES}

Argilli E, Sibley DR, Malenka RC, England PM, Bonci A (2008). Mechanism and time course of cocaine-induced long-term potentiation in the ventral tegmental area. J Neurosci 28: 9092-9100.

Barria A, Malinow R (2002). Subunit-specific NMDA receptor trafficking to synapses. Neuron 35: 345-353.

Borgland SL, Malenka RC, Bonci A (2004). Acute and chronic cocaine-induced potentiation of synaptic strength in the ventral tegmental area: electrophysiological and behavioral correlates in individual rats. J Neurosci 24: 7482-7490.

Brothwell SL, Barber JL, Monaghan DT, Jane DE, Gibb AJ, Jones S (2008). NR2B- and NR2D-containing synaptic NMDA receptors in developing rat substantia nigra pars compacta dopaminergic neurones. J Physiol 586: 739-750.

Brown TE, Lee BR, Mu P, Ferguson D, Dietz D, Ohnishi YN et al (2011). A silent synapse-based mechanism for cocaine-induced locomotor sensitization. J Neurosci 31: 8163-8174.

Chergui K, Akaoka H, Charlety PJ, Saunier CF, Buda M, Chouvet G (1994). Subthalamic nucleus modulates burst firing of nigral dopamine neurones via NMDA receptors. NeuroReport 5: 1185-1188.

Chuhma N, Choi WY, Mingote S, Rayport S (2009). Dopamine neuron glutamate cotransmission: frequency-dependent modulation in the mesoventromedial projection. Neuroscience 164: 1068-1083.

Cull-Candy S, Brickley S, Farrant M (2001). NMDA receptor subunits: diversity, development and disease. Curr Opin Neurobiol 11: 327-335.

Danbolt NC (2001). Glutamate uptake. Prog Neurobiol 65: 1-105.

Faleiro LJ, Jones S, Kauer JA (2004). Rapid synaptic plasticity of glutamatergic synapses on dopamine neurons in the ventral tegmental area in response to acute amphetamine injection. Neuropsychopharmacology 29: 2115-2125.

Gubner NR, Phillips TJ (2015). Effects of nicotine on ethanolinduced locomotor sensitization: a model of neuroadaptation. Behav Brain Res 288: 26-32. 
Harnett MT, Bernier BE, Ahn KC, Morikawa H (2009). Bursttiming-dependent plasticity of NMDA receptor-mediated transmission in midbrain dopamine neurons. Neuron 62: 826-838.

Huang YH, Lin Y, Mu P, Lee BR, Brown TE, Wayman G et al (2009). In vivo cocaine experience generates silent synapses. Neuron 63: 40-47.

Isaac JT, Nicoll RA, Malenka RC (1995). Evidence for silent synapses: implications for the expression of LTP. Neuron 15: 427-434.

Johnson LA, Furman CA, Zhang M, Guptaroy B, Gnegy ME (2005). Rapid delivery of the dopamine transporter to the plasmalemmal membrane upon amphetamine stimulation. Neuropharmacology 49: 750-758.

Kahlig KM, Lute BJ, Wei Y, Loland CJ, Gether U, Javitch JA et al (2006). Regulation of dopamine transporter trafficking by intracellular amphetamine. Mol Pharmacol 70: 542-548.

Kalivas PW, Alesdatter JE (1993). Involvement of $N$-methyl-Daspartate receptor stimulation in the ventral tegmental area and amygdala in behavioral sensitization to cocaine. J Pharmacol Exp Ther 267: 486-495.

Karler R, Calder LD, Chaudhry IA, Turkanis SA (1989). Blockade of 'reverse tolerance' to cocaine and amphetamine by MK-801. Life Sci 45: 599-606.

Kelly MA, Low MJ, Rubinstein M, Phillips TJ (2008). Role of dopamine D1-like receptors in methamphetamine locomotor responses of D2 receptor knockout mice. Genes Brain Behav 7: 568-577.

Kerchner GA, Nicoll RA (2008). Silent synapses and the emergence of a postsynaptic mechanism for LTP. Nat Rev Neurosci 9: 813-825.

Koya E, Cruz FC, Ator R, Golden SA, Hoffman AF, Lupica CR et al (2012). Silent synapses in selectively activated nucleus accumbens neurons following cocaine sensitization. Nat Neurosci 15: 1556-1562.

Liu XY, Chu XP, Mao LM, Wang M, Lan HX, Li MH et al (2006). Modulation of D2R-NR2B interactions in response to cocaine. Neuron 52: 897-909.

Lominac KD, Sacramento AD, Szumlinski KK, Kippin TE (2012). Distinct neurochemical adaptations within the nucleus accumbens produced by a history of self-administered vs non-contingently administered intravenous methamphetamine. Neuropsychopharmacology 37: 707-722.

Mao LM, Wang W, Chu XP, Zhang GC, Liu XY, Yang YJ et al (2009). Stability of surface NMDA receptors controls synaptic and behavioral adaptations to amphetamine. Nat Neurosci 12: 602-610.

Overton PG, Clark D (1997). Burst firing in midbrain dopaminergic neurons. Brain Res Brain Res Rev 25: 312-334.

Robertson GS, Damsma G, Fibiger HC (1991). Characterization of dopamine release in the substantia nigra by in vivo microdialysis in freely moving rats. J Neurosci 11: 2209-2216.

Rosenmund C, Clements JD, Westbrook GL (1993). Nonuniform probability of glutamate release at a hippocampal synapse. Science 262: 754-757.

Saunders C, Ferrer JV, Shi L, Chen J, Merrill G, Lamb ME et al (2000). Amphetamine-induced loss of human dopamine transporter activity: an internalization-dependent and cocainesensitive mechanism. Proc Natl Acad Sci USA 97: 6850-6855.

Schilstrom B, Yaka R, Argilli E, Suvarna N, Schumann J, Chen BT et al (2006). Cocaine enhances NMDA receptor-mediated currents in ventral tegmental area cells via dopamine D5 receptor-dependent redistribution of NMDA receptors. J Neurosci 26: 8549-8558.

Schumann J, Matzner H, Michaeli A, Yaka R (2009). NR2A/Bcontaining NMDA receptors mediate cocaine-induced synaptic plasticity in the VTA and cocaine psychomotor sensitization. Neurosci Lett 461: 159-162.

Scimemi A, Tian H, Diamond JS (2009). Neuronal transporters regulate glutamate clearance, NMDA receptor activation, and synaptic plasticity in the hippocampus. J Neurosci 29: 14581-14595.
Sharp T, Zetterstrom T, Ljungberg T, Ungerstedt U (1987). A direct comparison of amphetamine-induced behaviours and regional brain dopamine release in the rat using intracerebral dialysis. Brain Res 401: 322-330.

Shen H, Moussawi K, Zhou W, Toda S, Kalivas PW (2011). Heroin relapse requires long-term potentiation-like plasticity mediated by NMDA2b-containing receptors. Proc Natl Acad Sci USA 108: 19407-19412.

Sobczyk A, Scheuss V, Svoboda K (2005). NMDA receptor subunitdependent $\left[\mathrm{Ca}^{2+}\right]$ signaling in individual hippocampal dendritic spines. J Neurosci 25: 6037-6046.

Stuber GD, Hnasko TS, Britt JP, Edwards RH, Bonci A (2010). Dopaminergic terminals in the nucleus accumbens but not the dorsal striatum corelease glutamate. J Neurosci 30: 8229-8233.

Sulzer D (2011). How addictive drugs disrupt presynaptic dopamine neurotransmission. Neuron 69: 628-649.

Sulzer D, Rayport S (1990). Amphetamine and other psychostimulants reduce $\mathrm{pH}$ gradients in midbrain dopaminergic neurons and chromaffin granules: a mechanism of action. Neuron 5: 797-808.

Tovar KR, McGinley MJ, Westbrook GL (2013). Triheteromeric NMDA receptors at hippocampal synapses. J Neurosci 33: 9150-9160.

Tovar KR, Westbrook GL (1999). The incorporation of NMDA receptors with a distinct subunit composition at nascent hippocampal synapses in vitro. J Neurosci 19: 4180-4188.

Tovar KR, Westbrook GL (2002). Mobile NMDA receptors at hippocampal synapses. Neuron 34: 255-264.

Underhill SM, Wheeler DS, Li M, Watts SD, Ingram SL, Amara SG (2014). Amphetamine modulates excitatory neurotransmission through endocytosis of the glutamate transporter EAAT3 in dopamine neurons. Neuron 83: 404-416.

Ungless MA, Whistler JL, Malenka RC, Bonci A (2001). Single cocaine exposure in vivo induces long-term potentiation in dopamine neurons. Nature 411: 583-587.

Vanderschuren LJ, Schmidt ED, De Vries TJ, Van Moorsel CA, Tilders FJ, Schoffelmeer AN (1999). A single exposure to amphetamine is sufficient to induce long-term behavioral, neuroendocrine, and neurochemical sensitization in rats. J Neurosci 19: 9579-9586.

Vezina P, Queen AL (2000). Induction of locomotor sensitization by amphetamine requires the activation of NMDA receptors in the rat ventral tegmental area. Psychopharmacology (Berl) 151: 184-191.

Wadiche JI, Arriza JL, Amara SG, Kavanaugh MP (1995). Kinetics of a human glutamate transporter. Neuron 14: 1019-1027.

Wheeler DS, Underhill SM, Stolz DB, Murdoch GH, Thiels E, Romero G et al (2015). Amphetamine activates Rho GTPase signaling to mediate dopamine transporter internalization and acute behavioral effects of amphetamine. Proc Natl Acad Sci USA 112: E7138-E7147.

Wolf ME, Xue CJ, Li Y, Wavak D (2000). Amphetamine increases glutamate efflux in the rat ventral tegmental area by a mechanism involving glutamate transporters and reactive oxygen species. J Neurochem 75: 1634-1644.

Yap JJ, Covington HE III, Gale MC, Datta R, Miczek KA (2005). Behavioral sensitization due to social defeat stress in mice: antagonism at mGluR5 and NMDA receptors. Psychopharmacology (Berl) 179: 230-239.

Zhang XF, Hu XT, White FJ, Wolf ME (1997). Increased responsiveness of ventral tegmental area dopamine neurons to glutamate after repeated administration of cocaine or amphetamine is transient and selectively involves AMPA receptors. J Pharmacol Exp Ther 281: 699-706.

Zhang Y, Loonam TM, Noailles PA, Angulo JA (2001). Comparison of cocaine- and methamphetamine-evoked dopamine and glutamate overflow in somatodendritic and terminal field regions of the rat brain during acute, chronic, and early withdrawal conditions. Ann NY Acad Sci 937: 93-120. 